\title{
16
}

\section{An Axiom-Based Test Case Selection Strategy for Object-Oriented Programs*}

\author{
T.H. Tse ${ }^{\text {a }}$, F.T. Chan ${ }^{\text {b }}$, H.Y. Chen ${ }^{\text {c }}$ \\ a Department of Computer Science, the University of Hong Kong, Pokfulam Road, Hong Kong. \\ Email: tse@csd.hku.hk. Tel: (+852) 859 2183. Fax: (+852) 5598447. \\ b School of Professional and Continuing Education, the University of Hong Kong, Pokfulam Road, \\ Hong Kong.
}

c Department of Computer Science, the University of Hong Kong, Pokfulam Road, Hong Kong. (On leave from Jinan University, Guangzhou, China.)

\begin{abstract}
Because of the growing importance of object-oriented programming, a number of testing approaches have been proposed. Frankl et al. propose the application of the functional approach, using algebraic specifications for the generation of test cases and the validation of methods. Given a specification, Frankl et al. propose that equivalent terms should give observably equivalent objects, and offer general heuristics on the selection of equivalent terms for testing. Their guidelines, however, are only supported by limited empirical results, do not have a theoretical basis, and provide no guarantee of effectiveness.

In this paper, we define the concept of a fundamental pair as a pair of equivalent terms which are formed by replacing all the variables on both sides of an axiom by normal forms. We prove that an implementation is consistent with respect to all equivalent terms if and only if it is consistent with respect to all fundamental pairs. In other words, the testing coverage of fundamental pairs is identical to that of all equivalent terms, and hence we need only concentrate on the testing of fundamental pairs. Our strategy is mathematically based, simple, and much more efficient. Furthermore, it underscores the usefulness of axiom-based specifications.
\end{abstract}

Keyword Codes: D.2.1; D.2.5; D.1.5

Keywords: algebraic specifications, functional testing, object-oriented

\section{INTRODUCTION}

Object-oriented programming is considered as an increasingly popular software development method for the 1990s. Since testing is one of the most quality critical and time-consuming phases of the software development process, it is important to investigate into the testing of object-oriented programs. As indicated in [1], the important change from the testing of conventional programs to the testing of object-oriented programs is that the latter programs are not necessarily executed in a predefined order. The sequence of invocation of methods in a class is not specified explicitly.

\footnotetext{
* This research is supported in part by a Research and Conference Grant of the University of Hong Kong.
} 
Operations are allowed to be called in any order once an object has been created. Methods across different classes can be combined in various ways. Furthermore, many new testing problems related to inheritance, polymorphism, overloading, encapsulation, aggregation, and state-dependent behavior have arisen [2]. It is generally accepted, therefore, that object-oriented program testing is quite different from the conventional counterpart.

A number of papers on the testing of object-oriented programs have been published [3-14]. Most of the papers discuss the nature of the problem and potential solutions, using approaches such as functional testing, structural testing [5,9], and state-based testing [13,14]. In particular, Frankl et al. $[4,7,8]$ propose the application of the functional approach $[15,16]$ (or black-box testing) to objectoriented programming. They propose the use of algebraic specifications both as a means of selecting test cases for objects and for validating the results of testing. Different test cases are generated from algebraic "terms" satisfying the axioms in the specification. A set of automatic tools have been developed integrating test case generation with the execution and verification of test results. Unfortunately, there is a fundamental problem in the theory behind the equivalence of terms, and the proposed heuristics for the selection of terms is only formulated from limited empirical results.

In this paper, we propose an improved theoretical foundation for Frankl's functional approach, and a more efficient strategy for selecting equivalent terms as test cases. Our strategy reduces the domain of test case selection from a polynomial function of the set of axioms to a linear function. On the other hand, we formally prove that the testing coverage is as good as that proposed by Frankl et al.

\section{A SUMMARY OF FRANKL'S FUNCTIONAL TESTING APPROACH}

In their functional testing approach, Frankl et al. use algebraic specifications [17-19] of abstract data types (ADTs) to specify classes in target programs. A series of operations on an ADT is known as a term (or called a "word" in Frankl et al.). Let $u_{1}$ and $u_{2}$ be two terms of an ADT. Let $s_{1}$ and $s_{2}$ be the respective sequences of operations in a given implementation. According to Frankl et al., one term is equivalent to another if and only if one can be transformed into the other using axioms as rewriting rules. The test suite $\left(s_{1}, s_{2}\right)$ reveals an error of the implementation if $u_{1}$ is equivalent to $u_{2}$ but the operation sequences $s_{1}$ and $s_{2}$ produce observationally different objects.

Based on the above idea, Frankl et al. constructed a set of testing tools called ASTOOT. First of all, it accepts an algebraic specification and an implementation of the given class. It then accepts a term $u_{1}$, and generates an equivalent term $u_{2}$. Test drivers are created automatically by a driver generator to check and execute the operation sequences $s_{1}$ and $s_{2}$ corresponding to $u_{1}$ and $u_{2}$, respectively. Finally, the results of the executions of $s_{1}$ and $s_{2}$ are compared. If they do not give the same observational result, an error is found.

In Frankl's approach, test cases are generated by a compiler and a simplifier. The compiler translates the two sides of each axiom of an algebraic specification into a pair of ADT trees as a transformation rule. A conditional axiom in the specification will give rise to a branch in the ADT tree. The simplifier inputs an original term $\mathrm{u}_{1}$ provided by the user, translates it into an ADT tree, and applies the transformation rules in the form of the pairs of ADT trees to obtain an equivalent term $u_{2}$.

Two important problems are raised: How do we select an original term to be input to the simplifier, and how do we select paths through the resulting ADT trees, in order to increase the likelihood of exposing errors? To explore these problems, Frankl et al. performed two case studies, testing erroneous implementations of priority queues and sorted lists. After running several thousand test cases, they recommended the following tentative guidelines on the generation of test cases:

(i) Select long original terms, with various ratios of different operations.

(ii) For the case of conditional axioms, choose a variety of parameters so that all possible paths in the ADT tree would be traversed. 


\section{EVALUATION OF FRANKL'S APPROACH}

There are a number of merits in Frankl's functional approach and the associated tools:

(a) An integrated set of automatic tools are provided for test case generation, test driver generation, test execution, and test checking.

(b) After running a program with a test case, we must examine the result of execution against the specification of the program. This so called oracle problem is a major concern in program testing. The use of algebraic specifications is an elegant solution.

(c) Frankl's approach takes a suite of methods as a test case, instead of taking an individual method. This concept is especially useful in object-oriented programming, where the sequence of events does not depend on a predefined calling method but on a suite of messages passing among objects. There are, however, some problems in Frankl's approach:

(i) The definition of the equivalence of terms has a fundamental problem. Consider, for example, the two terms "([5]/[1]/[4]/[2]). sorting" and "([4]/[5]/[2]/[1]). sorting" in a specification for bubble sort (see Example 1 in the next section). They are regarded by most people as equivalent, and they also produce equivalent results when implemented correctly. However, they cannot be transformed into one another by left-to-right rewriting rules. Furthermore, transformation using axioms as rewriting rules is uni-directional. Thus a term $u_{1}$ being equivalent to $u_{2}$ would imply that $u_{2}$ is not equivalent to $u_{1}$. Frankl's papers are based on this fallacious definition.

(ii) The guidelines on the selection of equivalent test cases are supported only by two empirical studies, do not have any theoretical basis, and hence provide no guarantee of effectiveness.

\section{AN AXIOM-BASED STRATEGY FOR SELECTING EQUIVALENT TEST CASES}

In this paper, we propose a simple mathematically-based strategy for selecting equivalent test cases.

An algebraic specification of ADTs consists of a syntax declaration and a semantic specification $[18,19]$. The syntax declaration lists the functions involved, plus their domains and co-domains, corresponding to the input and output variables of methods. The semantic specification consists of axioms which describe the behavioral properties of the functions. The following is an example of an algebraic specification. Bubble sort is chosen because it is familiar to most readers.

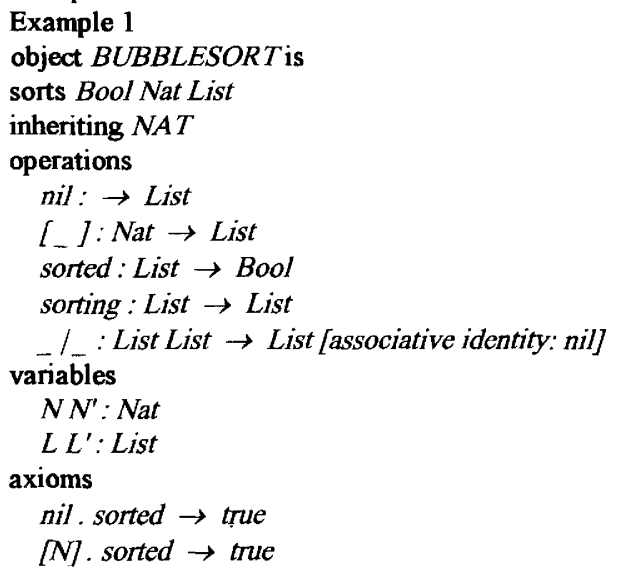




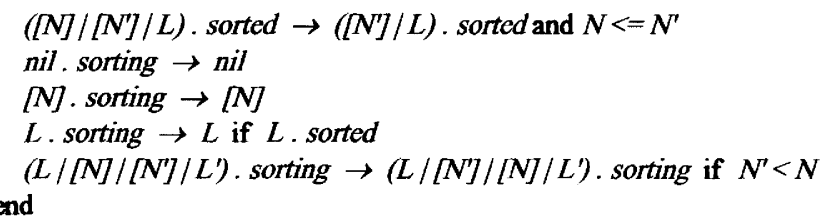

A list of functions on an ADT is called a term if and only if it conforms to the standard syntax requirements of term algebra [17, 18]. A term can be transformed into another using the axioms of the specification as progressive left-to-right rewriting rules. A term is said to be in normal form if and only if no further axiom is applicable. A system of axioms is said to be canonical if and only if every sequence of rewriting on the same ground term (that is, a term without variables) eventually reaches a unique normal form, independent of the choice and sequence of rewriting rules used. Thus, any consistent specification should be canonical.

The following is an improved version of the concept of equivalence:

Definition 4.1: Two terms $u_{1}$ and $u_{2}$ are said to be equivalent (denoted by $u_{1} \sim u_{2}$ ) if and only if both of them can be transformed by canonical axioms into the same normal form.

For instance, the terms "([5]/[1]/[4]/[2]). sorting" and "([4]/[5]/[2]/[1]). sorting" of Example 1 are equivalent because they can both be transformed into the same normal form $[1] /[2] /[4] /[5]$.

Furthermore, we would like to introduce a new concept in our paper:

Definition 4.2: Given a canonical system of axioms, a pair of equivalent terms, formed by replacing all the variables on both sides of an axiom by normal forms, is called a fundamental pair induced from the axiom.

The following two definitions are adapted from Frankl for completeness:

Definition 4.3: Given a canonical system of axioms, the series of methods corresponding to the functions in a term is called a sequence of operations corresponding to the term. Two such sequences $s_{1}$ and $s_{2}$ are equivalent (denoted by $s_{1} \approx s_{2}$ ) if and only if their operations on the same object give observationally equivalent results.

Definition 4.4: An implementation $\Psi$, which maps ADTs and functions of a canonical specification to classes and methods in a programming language, is said to be consistent with respect to the equivalent terms $u_{1} \sim u_{2}$ if and only if the corresponding sequences of operations satisfy $\Psi\left(u_{1}\right) \approx$ $\Psi\left(\mathrm{u}_{2}\right)$.

Having defined the fundamental concepts, we recommend a strategy for test case selection: in order to test whether an implementation is consistent with respect to equivalent terms, we need only test fundamental pairs. The testing coverage of fundamental pairs is the same as that of equivalent terms. In other words, any error revealable by equivalent terms can be revealed by some fundamental pair.

For instance, in Example 1, we need only test fundamental pairs such as

$$
([5] /[6] /[3] /[1] /[7] /[9]) \text {. sorting } \sim([5] /[6] /[1] /[3] /[7] /[9]) \text {. sorting }
$$
and $([1] /[3] /[5] /[6] /[7] /[9])$. sorting [1]/[3]/[5]/[6]/[7]/[9].

We need not test other equivalent pairs such as

$([6] /[5] /[3] /[1] /[9] /[7])$. sorting [1]/[3]/[5]/[6]/[7]/[9]

or $(([6] /[5])$. sorting $/([3] /[1])$. sorting /([9]/[7]). sorting). sorting

([5]/[6]/[3]/[1]/[7]/[9]). sorting.

Our strategy has a sound mathematical foundation, simple, and efficient. The testing coverage claim is based on Theorem 4.6 below. The following lemma is required in proving the theorem.

Lemma 4.5: Given a canonical specification, any term $u_{1}$ which is not in normal form can be transformed into a unique normal form $u_{0}$ via a series of axioms $a_{1}, a_{2}, \ldots, a_{k}$ : 


$$
\mathrm{u}_{1} \stackrel{\mathrm{a}_{1}}{\rightarrow} \mathrm{u}_{2} \stackrel{\mathrm{a}_{2}}{\rightarrow} \mathrm{u}_{3} \stackrel{\mathrm{a}_{3}}{\rightarrow} \quad \stackrel{\mathrm{a}_{\mathrm{k}}-1}{\rightarrow} \mathrm{u}_{\mathrm{k}} \stackrel{\mathrm{a}_{\mathrm{k}}}{\rightarrow} \mathrm{u}_{0}
$$

such that all the binding variables involved with each step of the transformation are in normal forms.

For example, the term "([5]/[1]/[4]/[2]) . sorting" of BUBBLESORT in Example 1 can be transformed into its normal form $[1] /[2] /[4] /[5]$ as follows:

$$
\begin{array}{llr} 
& ([5] /[1] /[4] /[2]) . \text { sorting } & \\
\rightarrow & ([5] /[1] /[2] /[4]) . \text { sorting } & \text { \{using the last axiom with } \\
& & \mathrm{L}=[5] \mid[1], \mathrm{N}=[4], \mathrm{N}= \\
\rightarrow & ([1] /[5] /[2] /[4]) . \text { sorting } & \\
\rightarrow & ([1] /[2] /[5] /[4]) . \text { sorting } & \\
\rightarrow & ([1] /[2] /[4] /[5]) . \text { sorting } & \\
\rightarrow & {[1] /[2] /[4] /[5]} &
\end{array}
$$

The proof of the general case is straightforward.

Theorem 4.6: For a given canonical specification, an implementation is consistent with respect to all equivalent terms if and only if it is consistent with respect to all fundamental pairs.

Proof of Theorem 4.6: Obviously if an implementation is consistent with respect to all equivalent terms, then it is consistent with respect to all fundamental pairs.

On the contrary, assume that an implementation is consistent with respect to all fundamental pairs. Let $u_{1} \sim u_{2}$ be any two equivalent terms. Suppose the mapping $\Psi$ denotes the implementation, and suppose $\Psi\left(\mathrm{u}_{1}\right)=\mathrm{s}_{1}$ and $\Psi\left(\mathrm{u}_{2}\right)=\mathrm{s}_{2}$. We would like to prove that $\mathrm{s}_{1} \approx \mathrm{s}_{2}$.

By Definition 4.1, $u_{1}$ and $u_{2}$ can be transformed into the same normal form $u_{0}$. Then, there exists a series of axioms $\mathrm{a}_{1}, \mathrm{a}_{2}, \ldots, \mathrm{a}_{\mathrm{k}}$ :

$$
\mathrm{u}_{1} \stackrel{\mathrm{a}_{1}}{\rightarrow} \mathrm{u}_{12} \stackrel{\mathrm{a}_{2}}{\rightarrow} \mathrm{u}_{13} \stackrel{\mathrm{a}_{3}}{\rightarrow} \ldots \stackrel{\mathrm{a}_{\mathrm{k}-1}}{\rightarrow} \mathrm{u}_{1 \mathrm{k}} \stackrel{\mathrm{a}_{\mathrm{k}}}{\rightarrow} \mathrm{u}_{0}
$$

satisfying Lemma 4.5 .

Let $\Psi\left(u_{1 j}\right)=s_{1 j}(j=2, \ldots, k)$ and $\Psi\left(u_{0}\right)=s_{0}$.

Suppose (without loss of generality) that *

(1) $u_{1}=f_{0}\left(A_{0}\right) \cdot f_{1}\left(A_{1}\right) \ldots f_{i}\left(A_{i}\right) \cdot f_{i+1}\left(A_{i+1}\right) \ldots f_{t}\left(A_{t}\right)$

where $A_{j}$ is either null or a tuple of parameters, containing only ground terms,

(2) $a_{1}$ is $O_{x} \cdot f_{1}\left(X_{1}\right) \ldots f_{i}\left(X_{i}\right) \rightarrow O_{x} \cdot g_{1}\left(Y_{1}\right) \ldots g_{j}\left(Y_{j}\right)$

where $\mathrm{O}_{\mathrm{X}}$ is null or an object variable, $\mathrm{X}_{2}, \ldots, \mathrm{X}_{\mathrm{i}}, \mathrm{Y}_{1}, \ldots, \mathrm{Y}_{\mathrm{j}}$ are either null or tuples of parameters containing variables and/or ground terms.

Then

$u_{12}=f_{0}\left(A_{0}\right) \cdot g_{1}\left(B_{1}\right) \ldots g_{j}\left(B_{j}\right) \cdot f_{i+1}\left(A_{i+1}\right) \ldots f_{t}\left(A_{t}\right)$

$s_{1}=\Psi\left(f_{0}\left(A_{0}\right)\right) \cdot \Psi\left(f_{1}\left(A_{1}\right)\right) \ldots \Psi\left(f_{i}\left(A_{i}\right)\right) . \Psi\left(f_{i+1}\left(A_{i+1}\right)\right) \ldots \Psi\left(f_{t}\left(A_{t}\right)\right)$

$s_{12}=\Psi\left(f_{0}\left(A_{0}\right)\right) \cdot \Psi\left(g_{1}\left(B_{1}\right)\right) \ldots \Psi\left(g_{j}\left(B_{j}\right)\right) . \Psi\left(f_{i+1}\left(A_{i+1}\right)\right) \ldots \Psi\left(f_{t}\left(A_{t}\right)\right)$

Since the series of axioms $a_{1}, \ldots, a_{k}$ satisfies Lemma 4.5,

${ }^{*}$ Consider, for example, the class of stacks. Suppose $\mathrm{u}_{1}$ in (1) is new . push(4) . pop . push(2). Then $\mathrm{f}_{0}\left(\mathrm{~A}_{0}\right)=$ new, $\mathrm{f}_{1}\left(\mathrm{~A}_{1}\right)=\operatorname{push}(4), \mathrm{f}_{2}\left(\mathrm{~A}_{2}\right)=$ pop, $\mathrm{f}_{3}\left(\mathrm{~A}_{3}\right)=\operatorname{push}(2)$. Suppose $\mathrm{a}_{1}$ in $(2)$ is $O_{X} \cdot \operatorname{push}(I)$. $p o p \rightarrow O_{X}$. Then $O_{X}$ matches new, push $(I)$ matches push( 4$)$, and pop matches pop. Hence, $\mathrm{u}_{12}=$ new . push(2). 
$f_{0}\left(A_{0}\right) \cdot f_{1}\left(A_{1}\right) \ldots f_{i}\left(A_{i}\right) \sim f_{0}\left(A_{0}\right) \cdot g_{1}\left(B_{1}\right) \ldots g_{j}\left(B_{j}\right)$

is a fundamental pair induced from $\mathrm{a}_{1}$. According to the assumption that the implementation is correct with respect to all fundamental pairs, we have

$\Psi\left(f_{0}\left(A_{0}\right)\right) . \Psi\left(f_{1}\left(A_{1}\right)\right) \ldots \Psi\left(f_{i}\left(A_{i}\right)\right) \approx \Psi\left(f_{0}\left(A_{0}\right)\right) . \Psi\left(g_{1}\left(B_{1}\right)\right) \ldots \Psi\left(g_{j}\left(B_{j}\right)\right)$.

Thus,

$\Psi\left(f_{0}\left(A_{0}\right)\right) \cdot \Psi\left(f_{1}\left(A_{1}\right)\right) \ldots \Psi\left(f_{i}\left(A_{i}\right)\right) \cdot \Psi\left(f_{i+1}\left(A_{i+1}\right)\right) \ldots \Psi\left(f_{t}\left(A_{t}\right)\right)$

$\approx \Psi\left(f_{0}\left(A_{0}\right)\right) . \Psi\left(g_{1}\left(B_{1}\right)\right) \ldots \Psi\left(g_{j}\left(B_{j}\right)\right) . \Psi\left(f_{i+1}\left(A_{i+1}\right)\right) \ldots \Psi\left(f_{t}\left(A_{t}\right)\right)$

i.e., $\mathrm{s}_{1} \approx \mathrm{s}_{12}$. By the same argument, $\mathrm{s}_{12} \approx \mathrm{s}_{13} \approx \ldots \approx \mathrm{s}_{1 \mathrm{k}} \approx \mathrm{s}_{0}$. Therefore, $\mathrm{s}_{1} \approx \mathrm{s}_{0}$.

Similarly we can prove that $\mathrm{s}_{2} \approx \mathrm{s}_{0}$. Hence $\mathrm{s}_{1} \approx \mathrm{s}_{2}$.

\section{CONTRIBUTIONS OF OUR STRATEGY}

Compared with Frankl's guidelines for selecting equivalent test cases, our strategy has the following advantages:

(a) Our strategy is based on a sound theoretical foundation and mathematical proof.

(b) The domain of test case selection in Frankl's guidelines is the whole set of all pairs of equivalent terms, but the domain of selection in our strategy is only the set of fundamental pairs induced from the axioms. The original domain is a polynomial function of the set of axioms, whereas the proposed domain is only a linear function of the set. Although our domain of selection is much less than that of Frankl's, our testing coverage is the same. Hence our strategy is more efficient. Furthermore, our strategy is more precise than Frankl's guidelines, and hence easier to be performed.

(c) Our recommendation is very natural since experienced implementors would start their tests with fundamental pairs even in the absence of formal theory. Our strategy simply says that when implementors are satisfied with the test results with respect to fundamental pairs, they do not need to test other equivalent terms.

(d) Given an original term $u_{1}$, Frankl's approach searches and reduces an ADT tree to generate a term $u_{2}$ such that $u_{1} \sim u_{2}$. "The size of the tree may be exponential in the size of the initial sequence. To deal with this complexity, the simplifier can operate either in batch mode, which builds the entire tree, or in interactive mode, which allows the user to selectively guide the construction of a subtree." [7] The interactive method can only give partial solution, whereas the batch method simply hides the complexity problem from the user. Our strategy avoids the complexity problem entirely by generating equivalent test cases directly from the two sides of each axiom, rather than searching and reducing the ADT trees.

(e) Using our strategy, two of Frankl's tools, the compiler and simplifier, can be replaced by a simple generator which induces equivalent test cases directly from the two sides of each axiom. This will greatly simplify the system.

\section{CONCLUSION}

In this paper, we take a fresh look at Frankl's functional approach for the testing of object-oriented programs. We find that, although the approach has many general merits, it is based on a fallacious foundation, and the heuristics for the selection of equivalent test cases are supported only by two empirical studies. Hence the correctness and effectiveness of the guidelines are not guaranteed. We 
define the concept of a fundamental pair as a pair of equivalent terms which are formed by replacing all the variables on both sides of an axiom by normal forms. We prove that an implementation is consistent with respect to all equivalent terms if and only if it is consistent with respect to all fundamental pairs. In other words, the testing coverage of fundamental pairs is identical to that of all equivalent terms, and hence we need only concentrate on the testing of fundamental pairs. We have reduced the domain of test cases from a polynomial function of the set of axioms to a linear function. Our strategy is mathematically based, simple, and much more efficient. Furthermore, it underscores the usefulness of axiom-based specifications.

We note that there may be infinitely many fundamental pairs induced from the same axiom by assigning different normal forms to the variables. Exhaustive testing is of course impossible. How should we select the fundamental pairs, and how does the selection affect the testing coverage? How do we verify whether two resulting objects are observationally equivalent? We are investigating into the application of regularity and uniformity hypotheses as proposed in $[15,16]$ to further enhance our strategy.

\section{REFERENCES}

1. SMITH, M.D. and ROBSON, D.J.: 'A framework for testing object-oriented programs', Journal of Object-Oriented Programming, 1992, 5 (3): pp.45-53.

2. KUNG, C.H., GAO, J., HSIA, P., LIN, J. and TOYOSHIMA, Y.: 'Design recovery for software testing of object-oriented programs', Proc. IEEE Workshop on Reverse Engineering, IEEE Computer Society, Los Alamitos, California, 1993.

3. CHEATHAM, T.J. and MELLINGER, L.: 'Testing object-oriented software systems', Proc. 18th ACM Computer Science Conference (CSC '90), ACM Press, New York, 1990, pp. 161-165.

4. DOONG, R.-K. and FRANKL, P.G.: 'Case studies on testing object-oriented programs', Proc. 4th ACM Annual Symposium on Software Testing, Analysis, and Verification (TAV 4), ACM Software Engineering Notes, 1991, pp. 165-177.

5. FIEDLER, S.P.: 'Object-oriented unit testing', Hewlett-Packard Journal, 1989, 40 (4): pp.69-74.

6. FIRESMITH, D.G.: 'Testing object-oriented software', in Technology of Object-Oriented Languages and Systems: Proceedings of 11th International Conference (TOOLS 11), PrenticeHall, Englewood Cliffs, New Jersey, 1993, pp. 407-426.

7. FRANKL, P.G. and DOONG, R.-K.: 'Tools for testing object-oriented programs', Proc. 8th Pacific Northwest Conference on Software Quality, 1990, pp. 309-324.

8. FRANKL, P.G.: 'A framework for testing object-oriented programs', Computer Science Technical Report PUCS-105-91, Department of Electrical Engineering and Computer Science, Polytechnic University, Brooklin, New York, 1991.

9. HARROLD, M.J., MCGREGOR, J.D. and FITZPATRICK, K.J.: 'Incremental testing of objectoriented class structures', Proc. 14th IEEE International Conference on Software Engineering, IEEE Computer Society, Los Alamitos, California, 1992, pp. 68-80.

10. HOFFMAN, D.M. and STROOPER, P.A.: 'Graph-based class testing', Proc. 7th Australian Software Engineering Conference (ASWEC '93), Australian Computer Society, 1993.

11. PERRY, D.E. and KAISER, G.E.: 'Adequate testing and object-oriented programming', Journal of Object-Oriented Programming, 1990, 3 (5): pp.13-19.

12. SMITH, M.D. and ROBSON, D.J.: 'Object-oriented programming: the problems of validation', Proc. 6th International Conference on Software Maintenance, IEEE Computer Society, Los Alamitos, Califormia, 1990, pp. 272-282.

13. TURNER, C.D. and ROBSON, D.J.: 'State-based testing and inheritance', Technical Report TR1/93, Computer Science Division, School of Engineering and Computer Science, University of Durham, Durham, UK, 1993. 
14. TURNER, C.D. and ROBSON, D.J.: 'Guidance for the testing of object-oriented programs', Technical Report TR-2/93, Computer Science Division, School of Engineering and Computer Science, University of Durham, Durham, UK, 1993.

15. BOUGE, L., CHOGUET, N., FRIBOURG, L. and GAUDEL, M.-C.: 'Test sets generation from algebraic specifications using logic programming', Journal of Systems and Software, 1986, 6: pp.343-360.

16. BERNOT, G., GAUDEL, M.-C. and MARRE, B.: 'Software testing based on formal specifications: a theory and a tool', Software Engineering Journal, 1991, 6 (6): pp.387-405.

17. EHRIG, H. and MAHR, B.: 'Fundamentals of Algebraic Specifications 1: Equations and Initial Semantics', EATCS Monographs on Theoretical Computer Science, 1985, Vol. 6, SpringerVerlag, Berlin.

18. GOGUEN, J.A., THATCHER, J.W. and WAGNER, E.G.: 'An initial algebra approach to specification, correctness and implementation of abstract data types', in Current Trends in Programming Methodology, Vol. IV: Data Structuring, R.T. Yeh (ed.), Prentice-Hall, Englewood Cliffs, New Jersey, 1978, pp. 80-149.

19. GOGUEN, J.A., WINKLER, T., MESEGUER, J., FUTATSUGI, K. and JOUANNAUD, J.-P.: 'Introducing OBJ3', in Applications of Algebraic Specification using OBJ, J.A. Goguen, D. Coleman, and R.M. Gallimore (eds.), Cambridge University Press, Cambridge, 1993.

Notice that a conditional axiom will generate several different fundamental pairs corresponding to different conditions. For instance, in the last axiom of Example 1,

$$
\begin{aligned}
L /[N] /\left[N^{\prime}\right] / L^{\prime} \text {. sorting } \rightarrow & \text { if } L /[N] /\left[N^{\prime}\right] / L^{\prime} \text {. sorted then } L /[N] /\left[N^{\prime}\right] / L^{\prime} \\
& \text { else if } N^{\prime}<N \text { then } L /\left[N^{\prime}\right] /[N] / L^{\prime} \text {. sorting }
\end{aligned}
$$

if we take $\mathrm{L}=[1], \mathrm{N}=3, \mathrm{~N}^{\prime}=5, \mathrm{~L}^{\prime}=[7]$, then we obtain the fundamental pair:

$[1] /[3] /[5] /[7]$. sorting $\sim[1] /[3] /[5] /[7]$

On the other hand, if we take $\mathrm{L}=[5], \mathrm{N}=3, \mathrm{~N}=1, \mathrm{~L}^{\prime}=[7]$, we obtain another pair:

$[5] /[3] /[1] /[7]$. sorting $\sim[5] /[1] /[3] /[7]$. sorting 